\title{
Seroprevalence and Infection Attack Rate of COVID- 19 in Indian Cities
}

Daihai He ( $\nabla$ daihai.he@polyu.edu.hk)

The Hong Kong Polytechnic University

\section{Yiming Fei}

The Hang Seng University

\section{Shi Zhao}

Chinese University of Hong Kong

\section{Hainan Xu}

McMaster University

\section{Xingyue Zhang}

The Hong Kong Polytechnic University

\section{Salihu S Musa}

The Hong Kong Polytechnic University

\section{Research Article}

Keywords: COVID-19, pandemic, seroprevalence, attack rate, mathematical modelling

Posted Date: September 29th, 2021

DOl: https://doi.org/10.21203/rs.3.rs-934038/v1

License: (c) (i) This work is licensed under a Creative Commons Attribution 4.0 International License.

Read Full License 
4 Daihai $\mathrm{He}^{1, \#}$, Yiming $\mathrm{Fei}^{2}$, Shi Zhao ${ }^{3,4}$, Hainan $\mathrm{Xu}^{5}$, Xingyue Zhang ${ }^{1}$, and Salihu S Musa ${ }^{1,6}$ 5

61 Department of Applied Mathematics, The Hong Kong Polytechnic University, Hong Kong, 7 China

82 Department of math and stats, The Hang Seng University, Hong Kong, China

93 JC School of Public Health and Primary Care, Chinese University of Hong Kong, Hong

10 Kong, China

114 CUHK Shenzhen Research Institute, Shenzhen, China

125 Department of Math and Stats, McMaster University, Canada

136 Department of Mathematics, Kano University of Science and Technology, Wudil, Nigeria

15 Email of all authors:

16 DH: daihai.he@polyu.edu.hk;

17 YMF: s196222@hsu.edu.hk

18 SZ: zhaoshi.cmsa@gmail.com;

19 HNX: xuh102@mcmaster.ca

20 XYZ: xingyue.zhang@connect.polyu.hk

21 SSM: salihu-sabiu.musa@connect.polyu.hk

23 Corresponding author:

24 \# DH: daihai.he@polyu.edu.hk 


\section{Abstract}

27 Objectives. Serological surveys were used to infer the infection attack rate in different 28 populations. The sensitivity of the testing assay, Abbott, drops fast over time since infection which make the serological data difficult to interpret. In this work, we aim to solve this issue.

Methods. We collect longitudinal serological data of Abbott to construct a sensitive decay function. We use the reported COVID-10 deaths to infer the infections, and use the decay function to simulate the seroprevalence and match to the reported seroprevalence in 12 Indian cities.

Results. Our model simulated seroprevalence match the reported seroprevalence in most (but not all) of the 12 Indian cities we considered. We obtain reasonable infection attack rate and infection fatality rate for most of the 12 Indian cities.

Conclusions. Using both reported COVID-19 deaths data and serological survey data, we infer the infection attack rate and infection fatality rate with increased confidence.

42

Keywords: COVID-19; pandemic; seroprevalence; attack rate; mathematical modelling.

\section{Introduction}

The COVID-19 pandemic has caused tremendous impact globally. The situation in

49 terms of reported cases and deaths were low in the year 2020 in India. It was once claimed 
50 that the control in India was a success until a major second wave hit the country and causing

51 large number of mortalities. But the reported deaths per capita is still relatively low compared

52 to other Western countries. Velumani et al. (Velumani et al. 2021) studied seroprevalence in

5312 Indian cities from July to December 2020. Their result showed two distinct patterns: a

54 wave pattern, e.g., Mumbai and Pune, and a monotone increasing pattern, e.g. Delhi and

55 others. These patterns were driven by the underlying COVID-19 pandemic in each city.

56 However, the other key drivers are the use of multiple brands of antibody testing machines

57 (or assays): in particular Abbott and non-Abbott, e.g., Roche. Abbott and Roche are widely

58 used serological testing devices in the market. The method of Abbott SARS-CoV-2 IgG assay

59 is acridinium-labelled anti-human IgG. Roche used ruthenium-labelled and biotin-conjugated

$60 \mathrm{~N}$ protein to perform the Anti-SARS-CoV total antibody assay. Velumani et al. (Velumani et

61 al. 2021) highlighted that two machines were used in this study, without specifying which

62 city used which one. Presumably, the Abbott was used in these wave-pattern cities while

63 Roche was in others (we explain the reason later). The two machines have very different

64 'decay rates' as found in (Muecksch et al. 2021). With data from (Muecksch et al. 2021),

65 Figure 1 shows a comparison of the sensitivity of Abbott vs Roche.

We observed that Abbott showed a very rapid drop in sensitivity over time for RT-PCR confirmed infections after RT-PCR confirmation (or onset of symptom for symptomatic cases after RT-PCR confirmed). While the sensitivity of Roche is stable. Moreover, as explained in the method (later section). The seroprevalence obtained with Roche would be much closer to the infection attack rate (IAR, defined as the cumulative proportion of the infected population), which shows a monotone increasing pattern. Since the antibody level of infected individual will gradually wane over time which causing the sensitivity of the testing drop (an infected person became seronegative), the seroprevalence will be lower than the attack rate

74 (AR), and this is very evident with Abbott than Roche. We first estimate the sensitivity drop 
75 function of Abbott based on publicly available data of Abbott. Then we collect reported COVID-19 deaths and serological studies from 12 Indian cities. To connect the two sources of data, we use the independently estimate sensitivity decay function of Abbott to reconstruct the IAR and the seroprevalence simultaneously in 12 Indian cities. We found that our approach works well in most cities where Abbott was likely and failed in a few cities where non-Abbott was likely used. Since we combine two sources of data, our inference on the IAR should be of higher confidence than using a single source of data.

\section{Material and Methods}

\section{Data}

COVID-19 deaths data were obtained from https://api.covid19india.org. Serological survey data for 12 Indian cities were obtained from (Velumani et al. 2021). A serological survey is usually carried out to investigate the prevalence of the size of an epidemic (population infected) in a given population by identifying the presence of specific antibodies used against the virus (Anand et al. 2021; George et al. 2021; Inbaraj, George, and

Chandrasingh 2021; Murhekar et al. 2020; Murhekar, Bhatnagar, Selvaraju, et al. 2021;

Murhekar, Bhatnagar, Thangaraj, et al. 2021; Velumani et al. 2021).

\section{Method}

\section{Sensitivity decay of Abbott}

To quantify the drop in Abbott, we collected data from several independent studies

94 (Maine et al. 2020; Muecksch et al. 2021; Eberhardt et al. 2021; Kahre et al. 2021; Harris et al. 2021), pooled data together (see Figure 2a), and fitted a modified Gamma distribution function (which has a max value of 1 , thus not a distribution function anymore) to the data to

97 quantify the drop rate (see Figure $2 b$ ). Thus, $f(t)=\operatorname{Gamma}(t, \alpha, \beta)$. Hence, we find the 98 best estimate $\alpha=0.165, \beta=0.012$. 
Following (He et al. 2021), we denote the daily COVID-19 seroprevalence as $p$

(proportion of population showing seropositive / antibody positive at a given day), daily sero(sero-positive individual turns sero-negative due to antibody waning), then we have the following equation:

$$
p[T]=p[T-1]+p^{+}[\text {in the last } 24 \text { hours }]-p^{-}[\text {in the last } 24 \text { hours }]
$$

Assuming initially all these are zero, this can be written as

$$
p(T)=\sum_{0}^{T} p^{+}-\sum_{0}^{T} p^{-} .
$$

Denote $f(s)$ as the sensitivity decay function which is given in Figure $2 \mathrm{~b}$.

$$
p(T)=\int_{0}^{T} c(t) f(T-t) / N d t
$$

i.e., the convolution (here we rewrite the summation in the above equation into integral fashion) between $c(t)$ and $f(s)$, where $c(t)$ is the time series of daily new cases, $N$ is the size of the population. But, typically $c(t)$ is not very reliable due to time-varying testing policy and testing effort and a significant amount of asymptomatic cases. Nonetheless, the daily COVID-19 deaths are available ((by law in many regions) and relatively reliable than reported cases. We denote $r$ as the infection fatality ratio (IFR). Thus, the daily new cases are roughly given as $c(t)=d(t+14) / r$. We assume a delay between deaths and case confirmation (ie, symptom onset for symptomatic cases) of 14 days. The seroprevalence on day $T$ is given:

$$
p(T)=\int_{0}^{T} r^{-1} d(t+14) f(T-t) / N d t
$$

120 We use the black curve in Figure $2 \mathrm{~b}$ as $f(t)$ for Abbott. When $f(t) \equiv 1, p(T)=A R(T)$, the 121 infection attack rate is: 


$$
\operatorname{IAR}(T)=\int_{0}^{T} r^{-1} d(t+14) / N d t .
$$

123 Thus given $f(s)$ and death data, we can get $p(t)$ and $I A R(t)$ easily if $r$ is known. However, $r$

124 estimates vary in previous studies, across locations and rely on the efforts of COVID-19

125 cases and deaths reporting. We argue that we may estimate $r$ by matching $p(t)$ to reported

126 seroprevalences at different time points. If the matching is acceptable, then we may accept

127 the reported seroprevalences (given that it was Abbott based), and we immediately obtain the 128 IAR and $r$.

129 Results

Figure 3 and Table 1 shows the main results, where we applied our method to 12 large

131 Indian cities. Since we do not know the infection fatality ratio (IFR), we align the

132 reconstructed seroprevalence (blue dashed curve) and serological survey (black curve with 133 circles) to be equal on Oct 15, 2020; this alignment will yield an IFR $r$, which is applied to 134 the whole reconstructed seroprevalence and the IAR curve (red curve). The reconstructed and 135 observed seroprevalence matched reasonably well for most cities, where Abbott is likely used 136 for most of the period. This works fine for most cities except for a few cities, e.g.,

137 Ahmedabad. In Ahmedabad, the serological survey went up too fast than the increase in IAR.

138 Thus, some mechanism unknown might occur (e.g. non-Abbott or inconsistence assay or 139 inconsistence in death reports) which affected the serological survey or dramatic changing in 140 COVID-19 death reporting process.

141 Table 1. Summary of estimates of infection attack rate and seroprevalence for 12 Indian 142 cities.

In our method, we assumed a reported COVID-19 accurately reflect the attack rate 144 subject to a constant IFR. This could fail if the death reporting effort dramatically changes or 
the intrinsic IFR changes (likely due to the emergence of new variants). The reconstructed seroprevalence successfully matched with the reported serological survey in Mumbai, Delhi,

147 Chennai, and Coimbatore. The reconstructed seroprevalence also successfully matched with

148 the trend of serological surveys in Kolkata, Nagpur, and Visakhapatnam. The method failed 149 in the rest, where a non-Abbott (or change of testing assay) was likely used. We do not have 150 sufficient data to reconstruct the sensitivity decay curve of Roche. Once it is available, a similar approach can be made readily (we leave for further study). In Figures $1 \& 2$, we assume a peak sensitivity of $100 \%$ for Abbott, which could be too ideal (since the data collected was mainly symptomatic cases). For asymptomatic cases, the peak sensitivity could be as low as $80 \%$. However, as long as the shape of the decay curve is kept, a factor $(80 \%)$ on the whole decay curve can be translated into a factor in the IFR. The reciprocal of the IFR and the sensitivity decay rate appear in a product form in our IAR and seroprevalence equation. Therefore, it would not change our final estimate of IAR and seroprevalence when the factor is in IFR or in the decay rate.

Moreover, we formulated a simple method to reconstruct the IAR and estimate the seroprevalence of COVID-19 deaths in India. Our simple approach works well in most of 12 Indian cities where serological survey data are available. We achieved this without knowing which cities used Abbott (we know Abbott was used in Mumbai). In some cities where our method did not yield a reasonable fit were likely due to the use of a non-Abbott (e.g. Roche) or mixing of different testing assays. According to the available information, we observed that the serological testing equipment used in Mumbai is Abbott and Delhi used Mylab, German Altona Diagnostics, Roche, and ELISA. The devices use in Chennai include Abbott, Roche, and ELISA. The instruments used in Kolkata include Vitrros ECi, Ortho Clinical Diagnostics, Roche, and ELISA. The device Banglore used was the Elecsys Anti-SARSCoV-2 assay. The equipment used in Ahmedabad was Zydus Diagnostics. The devices Pune 
used include ELISA and Roche. The device used in Surat was the ICMR National Institute of

171 Virology. The devices Nagpur used include Roche and ELISA. The devices used in

172 Coimbatore include Siemens and Abbott. The devices used in Jaipur include ICMR and IgM

173 /IgG Duo Test Kit (SD, Biosensor, Republic of Korea). Nevertheless, the device used in

174 Visakhapatnam is unknown. An alternative explanation could be that the serological survey

175 or death reporting is inconsistent in some cities where our approach fails. Our method

176 provided a reasonable and likely more reliable estimate of IAR by March 2021 in 12 Indian

177 cities since it used both serological data and reported COVID-19 deaths.

\section{Discussion}

Sero-epidemiological studies are crucial in understanding the current and future

scenario of the COVID-19 epidemics. Based on some recent reports, the average

seroprevalence rate of COVID-19 in India has been meagre, which indicates that a significant proportion of the overall population is still susceptible to COVID-19 infection (Murhekar,

Bhatnagar, Selvaraju, et al. 2021). This further highlighted that the virus's transmission could likely continue in most cities in India until the herd immunity threshold is reached. The herd immunity can be achieved either by previous exposure to the virus or through vaccination (Britton, Ball, and Trapman 2020; Murhekar, Bhatnagar, Selvaraju, et al. 2021). However, the successful control depends on the individual susceptibility to infection, pre-existing immunity, and compliance of non-pharmaceutical interventions (NPIs) measures, which could most likely affect the required number of infected individuals for the herd immunity threshold to be reached at a time of point (Aguas et al. 2020; Lourenço et al. 2020;

191 Simoneaux and Shafer 2020; Mendez-Brito, El Bcheraoui, and Pozo-Martin 2021).

The IFR estimates in Table 1 are low. On one hand, many serological studies found that a very large proportion of the population has been infected. On the other hand, the 
reported deaths is low (may or may not be due to under reporting). These two factors led to a seemingly low IFR in India. Banaji (Banaji 2021) found a lower bound IFR in Mumbai at $0.1 \%$ with consideration of excess deaths (additional to reported COVID-deaths), which is not very far from our estimates here. Here we used reported COVID-19 deaths only, which could lead to a low IFR.

We estimated the seroprevalence rate, and reconstructed an IAR in 12 Indian cities and 200 found a reasonable seropositivity rate to SARS-CoV-2 in most cities. We showed that the seroprevalence rate was lower than the reconstructed IAR, which is a monotone-increasing function due to antibody waning. Since we do not know the infection fatality ratio, we aligned the reconstructed seroprevalence and serological survey to be equal in the middle point of the study period, e.g., Oct 15, 2020, to yield an IFR, which was applied to both the 205 reconstructed seroprevalence and the IAR curve. These results are in line with previous 206 estimates, which highlighted the seroprevalence of COVID-19 deaths to be high in large cities; as well as with a limited number of serological surveys in more randomly selected populations (George et al. 2021; Velumani et al. 2021).

A significant amount of the people infected with SARS-CoV-2 do not show symptoms, 210 yet they transmit the disease to other susceptible populations (Long et al. 2020; Oran and 211 Topol 2021; Tu et al. 2020). It has been reported that asymptomatic cases of COVID-19 could reach from $5 \%$ up to about $80 \%$ of all infections (Inbaraj, George, and Chandrasingh 2021; Yanes-Lane et al. 2020), especially for young and healthy individuals. In slums settings 214 like India, these estimates could reach up to more than $90 \%$ (George et al. 2021; Velumani et 215 al. 2021). Therefore, it is imperative to identify an infected individual at the early stage of the 216 infection and trace all possible contacts in order to break the transmission paths, and also to 217 prevent more rapid spread of the pandemic. However, recognizing an asymptomatically (and 
pre-symptomatic) infected individual is practically difficult (and sometimes impossible) since they don't seek medical attention, which helps to identify their exposure to the disease (Gao et al. 2021; Inbaraj, George, and Chandrasingh 2021). As a result, relevant authorities, including the WHO, did not recommend absolute reliance on the morbidity and mortality cases identified through RT-PCR since it is very likely to have potential under-reporting issues (Inbaraj, George, and Chandrasingh 2021). research should be done on population-based sero-epidemiological surveys to generate more data that would help design the most effective control strategies ('World Health Organization. Coordinated global research roadmap: 2019 novel coronavirus; March 2020. Geneva: World Health Organization,' 2020). In addition, sero-epidemiological studies can help estimate the percentage of the population still susceptible to SARS-CoV-2. It is also important to investigate how Anti-SARS-CoV could provide immunity to the virus (Inbaraj, George, and Chandrasingh 2021). Most of early serosurveys, including the nationwide serosurvey that considered 21 Indian states by the Indian Council of Medical Research (ICMR), estimated the seroprevalence of COVID-19 in India to be $0.73 \%$ between May-June 2020 (Inbaraj, George, and Chandrasingh 2021; Murhekar et al. 2020). Subsequently (i.e., around August-September 2020), most serosurveys estimated the seroprevalence of SARS-CoV-2 in India to be $7-10 \%$ (George et al. 2021; Inbaraj, George, and Chandrasingh 2021; Khan et al. 2020; Murhekar, Bhatnagar, Selvaraju, et al. 2021; Murhekar, Bhatnagar, Thangaraj, et al. 2021). In their recent serosurvey study, Murhekar et al. estimated the overall seroprevalence of SARS-CoV2392 in India to be more than 24\% (Murhekar, Bhatnagar, Thangaraj, et al. 2021). 
the virus). It also helps to identify more cases, especially asymptomatically infected individuals who do not usually show COVID-19 symptoms, to design the most effective control strategies to mitigate the epidemics. High estimation of seroprevalence of COVID-19 would indicate that most of the population have been infected, and the herd immunity threshold will likely be reached (George et al. 2021; Kwok et al. 2021). Hence, the control efforts should be emphasized on recovering for the damages caused by this deadly virus, which includes suspension of control and intervention programmes, immunization and primary health care services for other infectious diseases; as well as rebuilding for better socio-economic development.

Our study has some limitations. We employed serological data that used multiple and mixing testing machines (Abbott and non-Abbott, e.g. Roche) to reconstruct an IAR and estimate the seroprevalence of COVID-19 in large cities in India. The true situation is much complicated than what we have considered. This could likely affect the actual estimation of seroprevalence in these cities. Further, we considered only 12 Indian cities; thus, interpretation for the overall scenarios has to be done with caution since new epidemiological features of SAR-CoV-2 is being discovered every day. We rely on the confidence on the

258 COVID-19 death reporting.

\section{Conclusion}

The seroprevalence of COVID-19 in India did not usually alter by sex or age group.

This paper reconstructed the attack rate and estimated the seroprevalence of COVI-19 deaths in 12 large Indian cities. We found a reasonable seropositivity rate to SARS-CoV-2 in most cities, where Abbott was likely used. We recommend that further sero-epidemiological studies and improved COVID mortality reporting couple with different existing control strategies can effectively help in suppressing the COVID-19 morbidity and mortality in India 
and beyond. Although we did not generate new data, we provide a way to connect data from

267 different sources (COVID-19 deaths and serological survey) which were treated separately in

268 previous studies. Hence the overall confidence on these data is enhanced and potential

269 inconsistence can be revealed.

270 Declarations

271 -Ethics approval and consent to participate

272 Not applicable.

273 -Consent for publication

274 Not applicable.

275 -Availability of data and materials

276 Serological data used in this work were taken from the following references, Maine et al,

277 2020, Muecksch et al 2020, Eberhardt et al 2020, Kahre et al, 2020 Harris et al, 2020.

278 Velumani et al. 2021. The COVID-19 deaths data for Indian cities were obtained from

279 https://api.covid19india.org.

280 -Competing interests

281 The authors declare that they have no competing interests.

282 -Funding

283 The work described in this paper was partially supported by a grant from the Research Grants

284 Council of the Hong Kong Special Administrative Region, China (HKU C7123-20G).

285 -Authors' contributions (This statement must exactly match on Editorial submission system 286 and in the manuscript) 
287 All authors conceived the study, carried out the analysis, wrote the draft, revised the manuscript critically, and approved it for publishing.

289

290

291

\section{-Acknowledgements}

None.

\section{Reference}

Aguas, Ricardo, Rodrigo M Corder, Jessica G King, Guilherme Goncalves, Marcelo U Ferreira, and M Gabriela M Gomes. 2020. 'Herd immunity thresholds for SARS-CoV-2 estimated from unfolding epidemics', medRxiv.

Anand, Shuchi, Maria Montez-Rath, Jialin Han, LinaCel Cadden, Patti Hunsader, Russell Kerschmann, Paul Beyer, Scott D Boyd, Pablo Garcia, and Mary Dittrich. 2021. 'Estimated SARS-CoV-2 Seroprevalence in US Patients Receiving Dialysis 1 Year After the Beginning of the COVID-19 Pandemic', JAMA network open, 4: e2116572-e72.

Banaji, Murad. 2021. 'Estimating COVID-19 infection fatality rate in Mumbai during 2020', medRxiv.

Britton, Tom, Frank Ball, and Pieter Trapman. 2020. 'A mathematical model reveals the influence of population heterogeneity on herd immunity to SARS-CoV-2', Science, 369: 846-49.

Eberhardt, Kirsten Alexandra, Felix Dewald, Eva Heger, Lutz Gieselmann, Kanika Vanshylla, Maike Wirtz, Franziska Kleipass, Wibke Johannis, Philipp Schommers, and Henning Gruell. 2021. 'Evaluation of a New Spike (S)-Protein-Based Commercial Immunoassay for the Detection of Anti-SARS-CoV-2 IgG', Microorganisms, 9: 733.

Gao, Zhiru, Yinghui Xu, Chao Sun, Xu Wang, Ye Guo, Shi Qiu, and Kewei Ma. 2021. 'A systematic review of asymptomatic infections with COVID-19', Journal of Microbiology, Immunology and Infection, 54: 12-16.

George, Carolin Elizabeth, Leeberk Raja Inbaraj, Sindhulina Chandrasingh, and LP de Witte. 2021. 'High seroprevalence of COVID-19 infection in a large slum in South India; what does it tell us about managing a pandemic and beyond?', Epidemiology \& Infection, 149.

Harris, Ross J, Heather J Whitaker, Nick J Andrews, Felicity Aiano, Zahin Amin-Chowdhury, Jessica Flood, Ray Borrow, Ezra Linley, Shazaad Ahmad, and Lorraine Stapley. 2021. 'Serological surveillance of SARS-CoV-2: Six-month trends and antibody response in a cohort of public health workers', Journal of Infection, 82: 162-69.

He, Daihai, Guihong Fan, Xueying Wang, Yingke Li, and Zhihang Peng. 2021. 'The new SARSCoV-2 variant and reinfection in the resurgence of COVID-19 outbreaks in Manaus, Brazil', medRxiv: 21254281.

Inbaraj, Leeberk Raja, Carolin Elizabeth George, and Sindhulina Chandrasingh. 2021. 'Seroprevalence of COVID-19 infection in a rural district of South India: A populationbased seroepidemiological study', PloS one, 16: e0249247. 
Kahre, Elisabeth, Lukas Galow, Manja Unrath, Luise Haag, Judith Blankenburg, Alexander Dalpke, Christian Lück, Reinhard Berner, and Jakob Peter Armann. 2021. 'Kinetics and seroprevalence of SARS-CoV-2 antibodies-a comparison of 3 different assays', medRxiv.

Khan, S Muhammad Salim, Mariya Amin Qurieshi, Inaamul Haq, Sabhiya Majid, Arif Akbar Bhat, Sahila Nabi, Nisar Ahmad Ganai, Nazia Zahoor, Auqfeen Nisar, and Iqra Nisar Chowdri. 2020. 'Seroprevalence of SARS-CoV-2 specific IgG antibodies in District Srinagar, northern India-a cross-sectional study', PloS one, 15: e0239303.

Kwok, Kin On, Edward B McNeil, Margaret Ting Fong Tsoi, Vivian Wan In Wei, Samuel Yeung Shan Wong, and Julian Wei Tsz Tang. 2021. 'Will achieving herd immunity be a road to success to end the COVID-19 pandemic?', Journal of Infection, 83: 381-412.

Long, Quan-Xin, Xiao-Jun Tang, Qiu-Lin Shi, Qin Li, Hai-Jun Deng, Jun Yuan, Jie-Li Hu, Wei Xu, Yong Zhang, and Fa-Jin Lv. 2020. 'Clinical and immunological assessment of asymptomatic SARS-CoV-2 infections', Nature medicine, 26: 1200-04.

Lourenço, José, Francesco Pinotti, Craig Thompson, and Sunetra Gupta. 2020. 'The impact of host resistance on cumulative mortality and the threshold of herd immunity for SARS-CoV-2', medRxiv.

Maine, Gabriel N, Kriselle Maris Lao, Subhashree Mallika Krishnan, Olabisi Afolayan-Oloye, Seyedalireza Fatemi, Sandeep Kumar, Lindsay VanHorn, Ashley Hurand, Elizabeth Sykes, and Qian Sun. 2020. 'Longitudinal characterization of the IgM and IgG humoral response in symptomatic COVID-19 patients using the Abbott Architect', Journal of Clinical Virology, 133: 104663.

Mendez-Brito, Alba, Charbel El Bcheraoui, and Francisco Pozo-Martin. 2021. 'Systematic review of empirical studies comparing the effectiveness of non-pharmaceutical interventions against COVID-19', Journal of Infection.

Muecksch, Frauke, Helen Wise, Becky Batchelor, Maria Squires, Elizabeth Semple, Claire Richardson, Jacqueline McGuire, Sarah Clearly, Elizabeth Furrie, and Neil Greig. 2021. 'Longitudinal serological analysis and neutralizing antibody levels in coronavirus disease 2019 convalescent patients', The Journal of infectious diseases, 223: 389-98.

Murhekar, Manoj V, Tarun Bhatnagar, Sriram Selvaraju, Kiran Rade, V Saravanakumar, Jeromie Wesley Vivian Thangaraj, Muthusamy Santhosh Kumar, Naman Shah, R Sabarinathan, and Alka Turuk. 2020. 'Prevalence of SARS-CoV-2 infection in India: Findings from the national serosurvey, May-June 2020', Indian Journal of Medical Research, 152: 48.

Murhekar, Manoj V, Tarun Bhatnagar, Sriram Selvaraju, V Saravanakumar, Jeromie Wesley Vivian Thangaraj, Naman Shah, Muthusamy Santhosh Kumar, Kiran Rade, R Sabarinathan, and Smita Asthana. 2021. 'SARS-CoV-2 antibody seroprevalence in India, August-September, 2020: findings from the second nationwide household serosurvey', The Lancet Global Health, 9: e257-e66.

Murhekar, Manoj V, Tarun Bhatnagar, Jeromie Wesley Vivian Thangaraj, V Saravanakumar, Muthusamy Santhosh Kumar, Sriram Selvaraju, Kiran Rade, CP Girish Kumar, R Sabarinathan, and Alka Turuk. 2021. 'SARS-CoV-2 seroprevalence among the general population and healthcare workers in India, December 2020-January 2021', International journal of infectious diseases, 108: 145-55.

Oran, Daniel P, and Eric J Topol. 2021. 'The proportion of SARS-CoV-2 infections that are asymptomatic: a systematic review', Annals of internal medicine, 174: 655-62. 
Simoneaux, Richard, and Steven L Shafer. 2020. 'Can herd immunity save us from COVID19?', Asa Monitor, 84: 18-19.

Tu, Huilan, Sheng Tu, Shiqi Gao, Anwen Shao, and Jifang Sheng. 2020. 'Current epidemiological and clinical features of COVID-19; a global perspective from China', Journal of Infection, 81: 1-9.

Velumani, Arokiaswamy, Chaitili Nikam, Wilson Suraweera, Sze Hang Fu, Hellen Gelband, Patrick E Brown, Isaac Bogoch, Nico Nagelkerke, and Prabhat Jha. 2021. 'SARS-CoV-2 Seroprevalence in 12 Cities of India from July-December 2020', medRxiv.

'World Health Organization. Coordinated global research roadmap: 2019 novel coronavirus; March 2020. Geneva: World Health Organization,'. 2020.

https://www.who.int/publications/m/item/a-coordinated-global-research-roadmap. Yanes-Lane, Mercedes, Nicholas Winters, Federica Fregonese, Mayara Bastos, Sara PerlmanArrow, Jonathon R Campbell, and Dick Menzies. 2020. 'Proportion of asymptomatic infection among COVID-19 positive persons and their transmission potential: A systematic review and meta-analysis', PloS one, 15: e0241536. 
Figures

409

410

411 Figure 1. A comparison of sensitivity of Abbott vs Roche over time. Data from (Muecksch 412 et al. 2021). The sensitivity of Abbott drops faster over time.

413

414 Figure 2 Abbott sensitivity as a function of time after symptom onset for a group of PCR positive patients (panel a). We assume the date of symptom onset and the date of RT-PCR confirmation are close. We fit a model (see main text) to the observed data (panel b).

419 Figure 3. Simulated attack rate (red curve) and seroprevalence (blue dashed curve) over time 420 compared with serological surveys (black curve with circles) in 12 Indian cities (Velumani et 421 al. 2021). The attack rate (AR) is a monotone-increase function of time given its definition.

422 The seroprevalence is lower than the AR due to sensitivity which is less than $100 \%$ and 423 decays rapidly over time. Since we do not know the infection fatality ratio, we simply align 424 the reconstructed seroprevalence and serological survey to be equal on Oct 15, 2020 to yield 425 an IFR which is applied to both the reconstructed seroprevalence curve and the AR curve. 426 
Figures

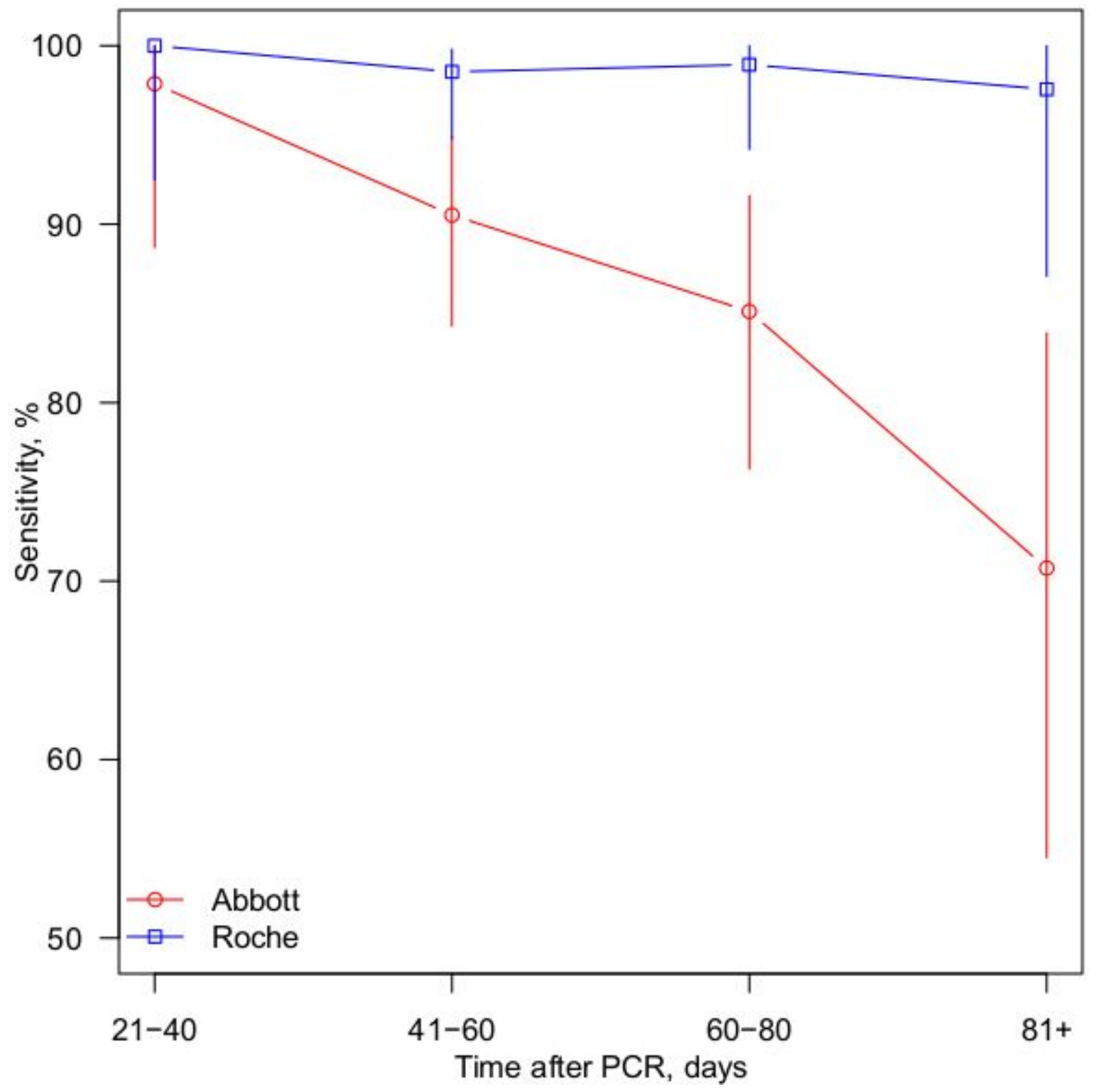

Figure 1

A comparison of sensitivity of Abbott vs Roche over time. Data from (Muecksch et al. 2021). The sensitivity of Abbott drops faster over time. 
a

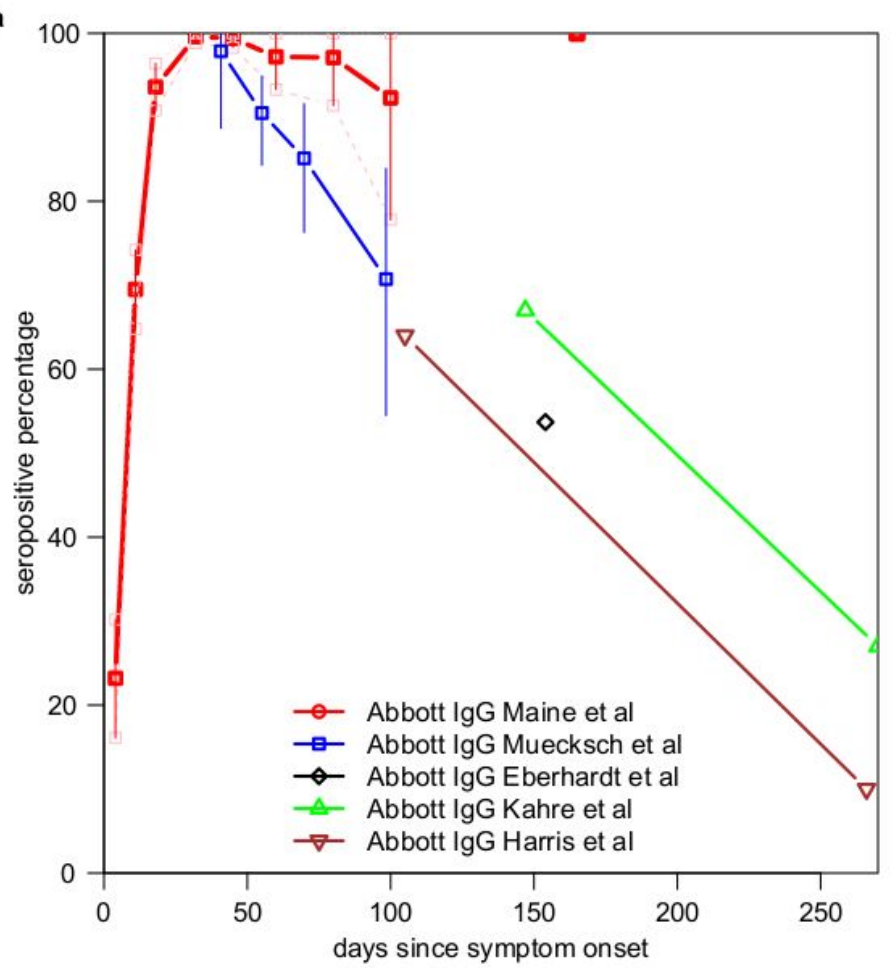

b

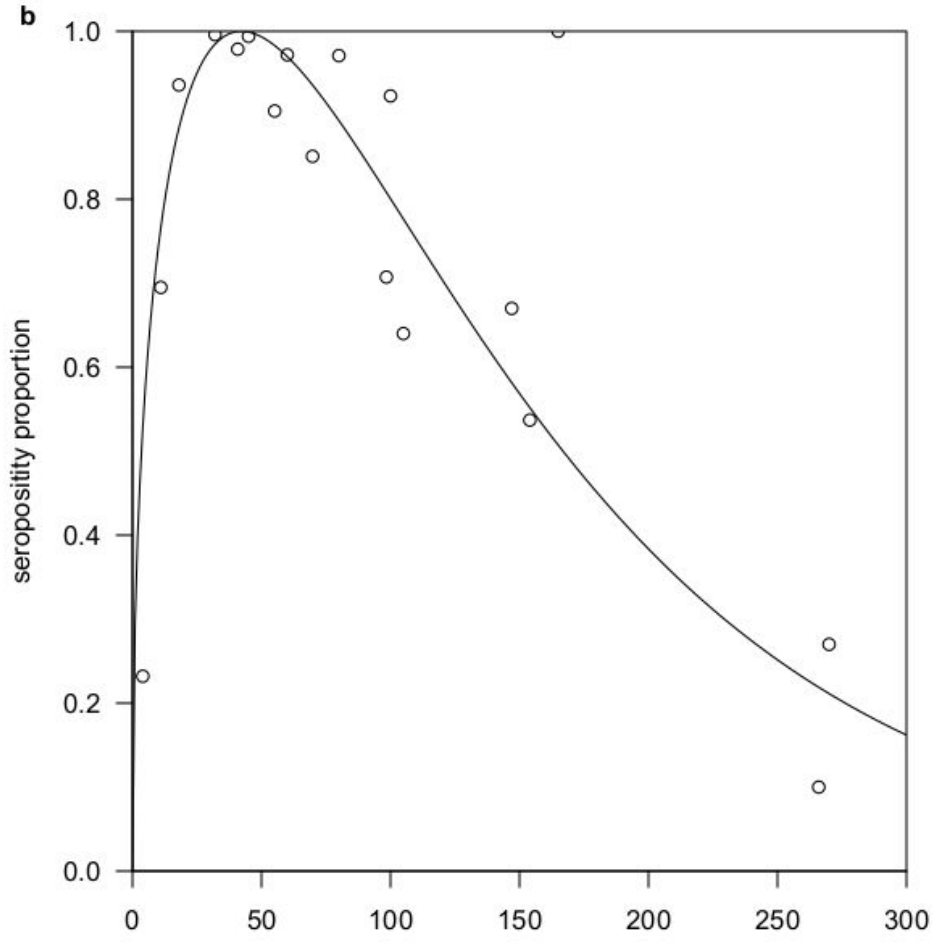

Figure 2

Abbott sensitivity as a function of time after symptom onset for a group of PCR positive patients (panel a). We assume the date of symptom onset and the date of RT-PCR confirmation are close. We fit a model (see main text) to the observed data (panel b). 

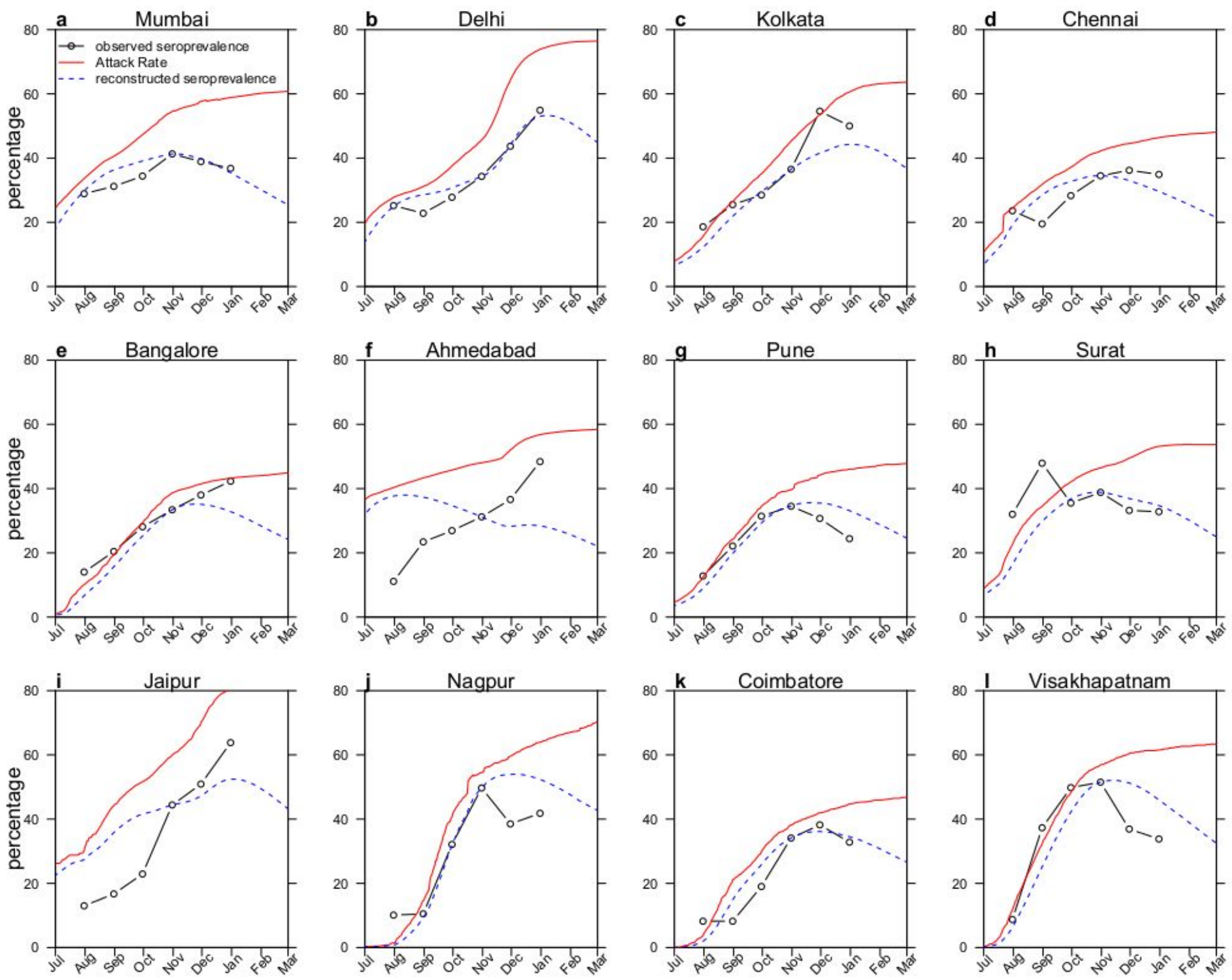

\section{Figure 3}

Simulated attack rate (red curve) and seroprevalence (blue dashed curve) over time compared with serological surveys (black curve with circles) in 12 Indian cities (Velumani et al. 2021). The attack rate $(A R)$ is a monotone-increase function of time given its definition. The seroprevalence is lower than the AR due to sensitivity which is less than $100 \%$ and decays rapidly over time. Since we do not know the infection fatality ratio, we simply align the reconstructed seroprevalence and serological survey to be equal on Oct 15, 2020 to yield an IFR which is applied to both the reconstructed seroprevalence curve and the AR curve. 